\title{
FCO3-95SF 20718
}

FINAL TECHNICAL REPORT

DOE/SF/207/8--T1

for DOE Grant

\section{"Krypton Spectroscopy Diagnosis of High Temperature Implosions"}

We carried out the proposed program as planned. The research work has resulted in several publications, see attachments. We addressed the questions from the statement of work in the proposal. It covered the topics of: (a) Observation of K-shell Krypton lines (b) Determination of temperature by continuous slope (c) Determination of temperature by line-intensity ratio (d) Mixing diagnosis based on high-opacity $\mathrm{Kr}$ lines (e) A method for measuring the escape factor (f) Diagnosis of density using low-opacity lines (g) Diagnosis of $\rho R$ using high-opacity lines.

A major program to demonstrate the feasibility of direct-drive laser fusion is underway at LLE. The overall goal of this program is to develop an understanding of target physics at the $30-\mathrm{kJ}$ level that will allow the performance of direct-drive capsules designed for use with the National Ignition Facility (NIF) at the 1- to 20-MJ level to be predicted with confidence. The majority of the experiments that will contribute to this goal will be performed with the $60 \mathrm{UV}$ (351-nm) beams of the recently upgraded, 30-kJ OMEGA laser system. The key physics issues that will be examined, both experimentally and theoretically, include irradiation uniformity, laser energy coupling and transport, laserplasma interaction physics, hydrodynamic stability, and hot-spot and main-fuellayer physics. In the upgrades OMEGA laser system, targets consisted mainly of glass microballoons (GMB's) having high initial aspect ratios ( $R / \Delta R$, where $R$ is the target radius and $\Delta \mathrm{R}$ is the shell thickness) of $\sim 150$ to 300 , filled with either pure deuterium or an equimolar mixture of deuterium and tritium at pressures 
ranging from 5 to $20 \mathrm{~atm}$. These low-mass shells were accelerated to high implosion velocities. The compressed fuel was predicted to have a high electron temperature (up to $6 \mathrm{keV}$ ) and a density in the range of 0.2 to $2 \mathrm{~g} / \mathrm{cm}^{3}$. Diagnosis of target performance during the acceleration phase of the implosion was obtained from time- and space-resolved measurements of the x-ray emission from the laser-heated glass shells. Overall target performance was diagnosed by nuclear and particle instrumentation, from which estimates of the total thermonuclear yield and the fuel ion temperature were obtained. Neutron yields have been measured to be as high as $10^{14}\left(\mathrm{D}^{\prime}\right)$ and $1.1 \times 10^{12}$ (DD), and ion temperatures up to $13 \mathrm{keV}$ have been inferred from a neutron time-of-flight detector. High fuel electron temperatures of 3 to $4 \mathrm{keV}$ have been inferred from spectroscopic measurements.

High-temperature implosion are planned for the OMEGA Upgrade experimental program. By using relatively thin shell targets, temperatures much higher than $1 \mathrm{keV}$ at modest compressed densities $\left(\sim 1\right.$ to $\left.5 \mathrm{~g} / \mathrm{cm}^{3}\right)$ are predicted. The goal of this work is to demonstrate that by adding a small admixture of krypton gas ( $~ 0.01 \mathrm{~atm})$ to the fuel, the temperature can be conveniently diagnosed through the spectrum of helium-like $\left(\mathrm{Kr}^{+34}\right)$ and hydrogen-like $\left(\mathrm{Kr}^{+35}\right)$ lines. By increasing the fill pressure, resonant $\mathrm{Kr}$ lines can become opaque, through self absorption, and their relative intensities can be used to diagnose shell-fuel mixing.

A simple way to estimate the expected intensity of krypton lines is to make a comparison with past experiments on argon-filled targets. In recent experiments on OMEGA, strong helium-like and hydrogen-like argon lines were observed when the argon fill pressure was $0.1 \mathrm{~atm}$ (in 20-atm deuterium). In other experiments the argon fill pressure was as low as 0.01 atm but still yielded 
significant spectral intensity. We chose to calculate the intensity of K-shell krypton lines using the corona approximation. This approximation was used to show only the intensity scaling; for the temperature-determination curves, the more general collisional-radiative model was used. The conclusion is that the strong K-shell krypton lines will be readily observable for temperatures $\geq 3 \mathrm{keV}$. Supportive evidence can be found in the fact that the resonance line of $\mathrm{Kr}^{+34}$ (at $0.94538 \AA$ ) was easily observable on previous short-pulse (100-ps) experiments on OMEGA, using a Von-Hamos focusing spectrometer. The peak laser power in those experiments was $\sim 6 \mathrm{TW}$, which is much lower than that of the OMEGA Upgrade ( 30 TW).

We then calculate the temperature dependence of a particular $\mathrm{Kr}$ lineintensity ratio under steady-state conditions, using the collisional-radiative atomic code POPION. For a temperature-sensitive line-intensity ratio we choose the ratio of a hydrogen-like line to a helium-like line. To minimize opacity effects we use the following two lines: (a) the Lyman- $\alpha$ line of $\mathrm{Kr}^{+35}$ of wavelength 0.9196 $\AA$, and (b) the helium- $\beta$ line of $\mathrm{Kr}^{+34}$ of wavelength $0.8033 \AA$ and absorption oscillator strength 0.1293 . We show the calculated intensity ratio of the Lyman- $\alpha$ line of $\mathrm{Kr}^{+35}$ to the helium- $\beta$ line of $\mathrm{Kr}^{+35}$ as a function of temperature for two electron-density values. Clearly, the intensity-ratio change is sensitive to temperature, but the Lyman- $\alpha$ line may be too weak to be observed for temperatures smaller than $\sim 4 \mathrm{keV}$. In going from $\mathrm{T}=10 \mathrm{keV}$ to $\mathrm{T}=4 \mathrm{keV}$, both the ratio and the intensity of the helium- $\beta$ line drop by an order of magnitude, which causes the intensity of the Lyman- $\alpha$ line to drop by two orders of magnitude. Over a wide density range (changing by a factor of 20 ), the temperature-dependence curve changes very little. To achive a $\pm 10 \%$ precision in the temperature, the intensity ratio must be measured with a precision of only a facotr of $\sim 3$ (at $T=4$ 
$\mathrm{keV}$ ) or a factor of $\sim 2$ (at $\mathrm{T}=6 \mathrm{keV}$ ).

Using the film and crystal calibrations, the spectral data were converted to spectral fluence in absolute units. The continuum slopes in the ranges 8 to $13 \mathrm{keV}$ (shot 4952 ) and 16.5 to $20.5 \mathrm{keV}$ (shot 5110) were used to estimate the electron temperature by fitting exponential curves. The result for shot 4952 shows a temperature of $3.1 \mathrm{keV}$ by fitting to the high-energy part of the continuum. For shot 5110 , the measured temperature was $4.0 \mathrm{keV}$. The simulated spectra show, in agreement with the experiments, that high-energy portions of the continuum yield higher temperatures. The experimental result for shot 4952 agrees with the simulated temperature of $3.2 \mathrm{keV}$, obtained from the slope of the simulated spectrum in the same spectral range. LILAC results show that the spaceaveraged temperatures around peak compression in these shots were 3 to $4 \mathrm{keV}$ and the maximum temperatures were 5 to $6 \mathrm{keV}$. The ion temperatures in such thin-shell implosions are predicted to be significantly higher than the electron temperatures, consistent with the experimental results reported.

Discussion shows that measuring the escape factor cannot yield information on the density or $\rho R$. Mixing of shell material into the fuel, however, does affect the escape factor. We can understand the effect of mixing on the opacity as follows: Without mixing, the opacity is approximately constant during the compression because of the two opposing effects: (1) increase in the $\rho R$ of absorbing ions and (2) increase in the linewidth. The mixed shell material is involved only in the second effect, which causes a net reduction in the opacity. The experimental determination of the mixing fraction consists of measuring the escape facotr. We can examine two experimental signatures: (a) for a uniform source, the core image size at high photon energy will be about the same as that at low photon energy, whereas for a hot-spot source the former will be much smaller than the latter; and (b) for a uniform source the observed line profile will be flat 
topped, whereas for a hot-spot source a self-reversal (or minimum) will be observed at the position of the profile peaks.

The escape factor of a line can be measured by comparing its measured intensity to that of another line, both of which have the same upper level. The first should have an opacity $\tau_{0} \gg 1$, the second $\tau_{0}<<1$. The two helium-like $\mathrm{Kr}$ lines we selected are (a) the Lyman- $\beta$ line, $1 \mathrm{~s} 3 \mathrm{p}{ }^{1} \mathrm{P}-1 \mathrm{~s}^{2}{ }^{1 \mathrm{~S}}$ (at $0.8033 \AA$ ) and (b) the Balmer- $\alpha$ line, 1s3p ${ }^{1} \mathrm{P}-1 \mathrm{~s} 2 \mathrm{~s}{ }^{1} \mathrm{~S}$ (at $5.0508 \AA$ ). By making an appropriate choice of the krypton fill pressure, the opacity (for resonant absorption) of the Lyman- $\beta$ line at peak compression will be $>>1$, while that of the Balmer- $\alpha$ will be $<<1$. In the absence of any absorption, the intensity ratio of these two lines would be given by the ratio of the Einstein A coefficients (spontaneous emission probabilities) and be independent of any atomic modeling. In the case discussed here, the observed intensity ratio will be lower than the ratio of the Einstein A coefficents, by the escape factor for the helium- $\beta$ line. We estimate the expected sensitivity of the method for measuring the degree of shell-fuel mixing. To find escape factor $G$ with a precision of approximately $\pm 20 \%$, the relative intensity of each of the lines must be measured with a precision of $\pm 10 \%$, which requires the relative calibration of two instruments for the two very differnt wavelengths used here.

Detailed $\mathrm{Kr}$ Stark profile improves and extends the earlier analysis. The use of krypton doping is a powerful technique for diagnosing target implosions, as the implosion dynamics are to lowest order independent of doping and different levels of doping can be used to permit the measurement of different quantities. For example, low doping levels allow low-opacity lines to be used to measure the ion temperature via Doppler broadening (they can provide a measurement of the ion temperature if spectrometers of spectral resolution $\Delta \lambda / \lambda \geq 1000$ are used), while higher doping levels allow the density-radius product $(\rho R)$ of the compressed fuel 
to be measured from high-opacity lines (through the determination of escape factor). In high-density implosions, low-opacity lines can be used to diagnose the density through Stark broadening, and higher-opacity lines can be used to estimate the extent of mixing. Calculations have been carried out using the LILAC code for a $\mathrm{CH}$ shell of $1-\mathrm{mm}$ diameter and $10-\mu \mathrm{m}$ thickness, filled with a 10-atm pressure of DT. The laser energy was $20 \mathrm{~kJ}$ in a Gaussian pulse of 650-ps width. Results have shown that the core electron temperature and density are fairly uniform, at $\sim 5 \mathrm{keV}$ and $\sim 4.5 \mathrm{~g} / \mathrm{cm}^{3}$, respectively. On the other hand, the ion temperature ranges from $\sim 6 \mathrm{keV}$ to $\sim 12 \mathrm{keV}$ and is centrally peaked. Code calculations have shown that the core temperatures are hardly changed by the addition of $0.01 \mathrm{~atm}$ of krypton. By adding $0.1 \mathrm{~atm}$ of krypton, the peak electron temperature drops a little to about $4 \mathrm{keV}$. The predicted spectra have been calculated by the LILAC code and a non-LTE post-processor, which includes transitions between actual atomic configurations, using Doppler and Stark profiles. The calculated intensities even for the 0.01-atm case are very substantial (of the order $10^{17} \mathrm{keV} / \mathrm{keV}$ ), and the contrast of line to continuum is better than a factor of 4 for the He- $\beta$ line. Furthermore, the shape of the spectrum above $\sim 10$ $\mathrm{keV}$ at peak compression differs little from the time-integrated spectrum because

most of the emission originates from peak compression. Thus, a time-integrated measurement in this spectral range yields the conditions at peak compression with reasonable reliability.

Attachments:

(1) "Krypton Spectroscopy Diagnosis of High-Temperature Implosions" LLE Review, Volume 61 
(2) "Initial Target Experiments on the Upgraded OMEGA Laser System" LLE Review, Volume 64

(3) "Diagnosis of High-Temperature Implosions Using Low- and High-Opacity Krypton Lines" LLE Review Volume 64

(4) "Diagnosis of High-Temperature Implosions Using Low- and High-Opacity Krypton Lines", B. Yaakobi, R. Epstein, C. F. Hooper, Jr., D. A. Hynes, Jr., and Q. Su, J. X-ray Sci. and Tech. (accepted, 1996).

\section{DISCLAIMER}

This report was prepared as an account of work sponsored by an agency of the United States Government. Neither the United States Government nor any agency thereof, nor any of their employees, makes any warranty, express or implied, or assumes any legal liability or responsibility for the accuracy, completeness, or usefulness of any information, apparatus, product, or process disclosed, or represents that its use would not infringe privately owned rights. Reference herein to any specific commercial product, process, or service by trade name, trademark, manufacturer, or otherwise does not necessarily constitute or imply its endorsement, recommendation, or favoring by the United States Government or any agency thereof. The views and opinions of authors expressed herein do not necessarily state or reflect those of the United States Government or any agency thereof. 